\title{
0 fever endocarditis: A surgical view and a word of caution
}

Thierry G. Mesana, MD, PhD, ${ }^{a, b}$ Frederic Collart, MD, ${ }^{b}$ Thierry Caus, MD, PhD, ${ }^{b}$ and Agnes Salamand, MD, ${ }^{\mathrm{b}}$ Marseille, France, ${ }^{\mathrm{b}}$ and Ottawa, Ontario, Canada ${ }^{\mathrm{a}}$

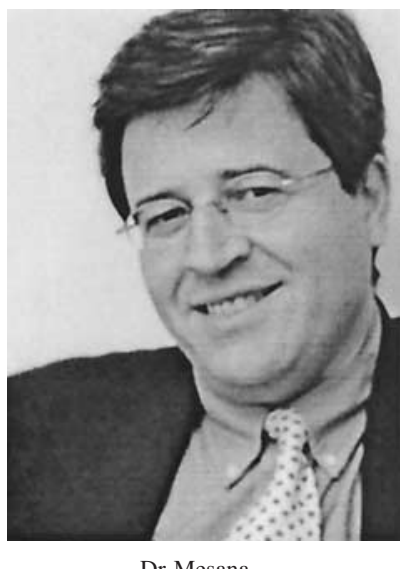

Dr Mesana

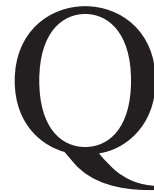
fever endocarditis is caused by Coxiella burnetii, which is linked to the Rickettsia family. This is a lethal but widely underestimated disease because of insidiousness and lack of knowledge, whereas it occurs worldwide, including North America. ${ }^{1-3}$ It is a highly resistant intracellular microorganism that is frequently involved in negative blood culture endocarditis, ${ }^{1,4}$ although it is easily detectable with a simple, low-cost, and reliable serologic examination with immunofluorescence. Because of a close relationship with a Nationwide Referral Centre for Rickettsioses, we have been developing surgical strategies that enabled us to more frequently identify $\mathrm{Q}$ fever in patients referred for valvular interventions with or without initial suspicion for endocarditis. We therefore observed, over a 15-year period, a consecutive series of 20 patients, representing, to our knowledge, the world's largest single surgical experience. ${ }^{5}$ This brief report aims to emphasize the role of the cardiac surgeon in this difficult-to-diagnose, difficultto-treat endocarditis.

\section{Clinical Summary}

Between May 1987 and October 2001, a total of 20 cases of Q fever endocarditis were diagnosed in our unit in 16 male and 4 female patients aged 36 to 79 years. Fever was reported in 13 patients and was discovered retrospectively. A peripheral embolic event revealed the diagnosis in 2 patients. As regards predisposition of the valve for endocarditis, we observed similar previous damage on aortic (9/20 [45\%]) versus mitral (11/20 [55\%]) valves and a similar ratio between native valves ( 9 patients) and prosthe-

\footnotetext{
From the Division of Adult Cardiac Surgery, University Hospital La Timone, Marseille, France.

Received for publication May 28, 2002; accepted for publication June 13, 2002.

Address for reprints: T. Mesana, MD, PhD, Chief, Division of Cardiac Surgery, University of Ottawa Heart Institute, 40, Ruskin Street, Ottawa, Ontario, Canada (E-mail: tmesana@ottawaheart.ca).

J Thorac Cardiovasc Surg 2003;125:217-8

Copyright (C) 2003 by The American Association for Thoracic Surgery

$0022-5223 / 2003 \$ 30.00+0$

doi: $10.1067 / \mathrm{mtc} .2003 .127$
}

ses (11 patients). Echocardiography mostly showed paraprosthetic leak or bioprosthetic deterioration with few signs of endocarditis, highlighting cardiac vegetation in only 4 patients and minimal abscess in 2 patients. Blood culture results remained negative in every patient. The diagnosis was assessed preoperatively in only 9 patients, suspected intraoperatively in 3 patients, and discovered postoperatively after routine bacterial analysis of valve specimens in 4 patients. In all instances, Coxiella burnetii was identified by means of indirect microimmunofluorescence on blood sampling, detecting antibodies directed against phase I and phase II antigens, with a serologic threshold of 1:800 immunoglobulin $\mathrm{G}(\mathrm{IgG})$ and 1:50 IgA. Our series showed 4 other patients having strictly normal functioning prostheses, which were successfully treated only with medication. We performed 8 mitral valve procedures (including 2 repairs), 7 aortic valve replacements (including 4 root replacements [3 homografts]), and a double valve replacement. All patients received prolonged oral specific antibiotics (doxycycline and hydroxychloroquine) for at least 18 months under serologic guidance. ${ }^{1,5}$ We observed no operative mortality, no relapses, and 2 noncardiac late deaths $(2 / 20[10 \%])$.

\section{Comment}

Coxiella burnetii endocarditis is considered rare, although it represents $3 \%$ to $5 \%$ endocarditis. ${ }^{1,4}$ In our hospital, where the Referral Center for Rickettsioses is situated, active Q fever was the most frequent cause in the presence of negative blood culture endocarditis, and the frequency increased with time and more aggressive search. We first performed serologic tests between 1987 and 1993 in the presence of clinical evidence of endocarditis, despite negative blood culture results, and then in the event of any unexplained inflammatory syndrome associated with native valve or prosthesis dysfunction and more recently (since 1999) for any valve-replacement procedure. Consequently, the disease was diagnosed and treated in 6 patients between 1987 and 1993, 7 patients between 1994 and 1998, and 7 patients in the last 3 years. Macroscopic surgical evaluation was nonspecific and favored endocarditis in only $50 \%$ of cases, retrieving little vegetation and rare abscesses. The most common surgical observation was paraprosthetic leak with limited inflammation of the leaflets or the anulus or the bioprosthetic cusps. Moreover, Q fever was often assessed through systematic search on valvular material, thus emphasizing the role of the surgeon requesting systematic valvular sample 
TABLE 1. Circumstances for $\mathbf{0}$ fever investigation in cardiac surgery

1. Preoperative circumstances

A. Any negative blood culture endocarditis

B. Isolated inflammatory syndrome with native-prosthetic valve

C. Cardiac failure with exposure to farm or pet animals

2. Intraoperative circumstances

A. Unexplained inflammatory aspect of native-prosthetic valve

B. Unexpected or repeated paravalvular leaks requiring reoperations

C. Early-unexpected bioprosthetic cusp deterioration

analysis, particularly with patients submitted to repeated procedures for paravalvular leakage. We also observed that valve culture results were positive, even when medication had started before the operation. This underlines the particular resistance of the bacterium because of intracellular development and the importance of the surgical eradication, followed by long-term medical treatment under serologic guidance. Indeed, a difficult issue arises when active $\mathrm{Q}$ fever is demonstrated with normal prosthesis function, and medication alone can be proposed. Three of our medically treated patients were operated on during follow-up for regular structural deterioration, although earlier replacement should be indicated in any event as minimal paraprosthetic leak or acceler- ated dysfunction. Finally, our low mortality rate and the absence of relapses showed the benefit of our strategy.

\section{Conclusion}

Q fever might often go undetected in geographic low-risk zones, such as North America, although the lack of knowledge might be catastrophic everywhere. More aggressive search is recommended in a variety of situations, as shown in Table 1, including systematic analysis of surgical specimen obtained during valve procedures.

We thank J. P. Casalta, MD, and D. Raoult, MD, Director of the French National Center for Rickettsiae, for their long-lasting and fruitful cooperation, worldwide expertise, and friendship in Hospital La Timone, Marseille, France.

\section{References}

1. Raoult D, Marrie TJ. Q fever. Clin Infect Dis. 1995;20:489-96.

2. Haldane E, Marrie TJ, Faulkner RS, Lee SH, Cooper JH, Mac Pherson DD, et al. Endocarditis due to Q fever in Nova Scotia: experience with five patients in 1981-1982. J Infect Dis. 1983;148:978-85.

3. Siegman Igra Y, Kaufman O, Keysary O. Q fever endocarditis in Israel and a worldwide rewiew. Scand J Infect Dis. 1997;29:41-9.

4. Fournier PE, Casalta JP, Habib G, Mesana T, Raoult D. Modification of the diagnostic criteria proposed by the Duke endocarditis service to permit improved diagnosis of Q fever endocarditis. Am J Med. 1996; 100:629-33.

5. Salamand A, Collart F, Caus T, Casalta JP, Mouly-Bandini A, Monties $\mathrm{JR}$, et al. Q fever endocarditis: over 14 years of surgical experience in a referal Center for Rickettsioses. J Heart Valve Dis. 2002;11:84-90. 